\title{
Ergodicity, rejuvenation, enhancement, and slow relaxation of diffusion in biased continuous-time random walks
}

\author{
Takuma Akimoto, ${ }^{1, *}$ Andrey G. Cherstvy, ${ }^{2}$ and Ralf Metzler ${ }^{2}$ \\ ${ }^{1}$ Department of Physics, Tokyo University of Science, Noda, Chiba 278-8510, Japan \\ ${ }^{2}$ Institute for Physics \& Astronomy, University of Potsdam, 14476 Potsdam-Golm, Germany
}

(Received 17 March 2018; revised manuscript received 12 May 2018; published 7 August 2018)

\begin{abstract}
Bias plays an important role in the enhancement of diffusion in periodic potentials. Using the continuous-time random walk in the presence of a bias, we report on an interesting phenomenon for the enhancement of diffusion by the start of the measurement in a random energy landscape. When the variance of the waiting time diverges, in contrast to the bias-free case, the dynamics with bias becomes superdiffusive. In the superdiffusive regime, we find a distinct initial ensemble dependence of the diffusivity. Moreover, the diffusivity can be increased by the aging time when the initial ensemble is not in equilibrium. We show that the time-averaged variance converges to the corresponding ensemble-averaged variance; i.e., ergodicity is preserved. However, trajectory-to-trajectory fluctuations of the time-averaged variance decay unexpectedly slowly. Our findings provide a rejuvenation phenomenon in the superdiffusive regime, that is, the diffusivity for a nonequilibrium initial ensemble gradually increases to that for an equilibrium ensemble when the start of the measurement is delayed.
\end{abstract}

DOI: 10.1103/PhysRevE.98.022105

\section{INTRODUCTION}

Mixing of more than two fluids is a key operation of microfluidic devices in chemistry, biology, and industry, in which diffusion is an essential mechanism for mixing [1-3]. In particular, achieving an enhancement of the diffusivity is pivotal for mixing of particles in heterogeneous environments because diffusion in such systems is often slow. One of the most applicable controls of the diffusivity is adding a directed external force or a flow, i.e., a bias. In discrete-time random walks the bias, characterized by the difference between the probabilities of right and left jumps, suppresses the diffusivity. In particular, the variance of the displacement grows linearly with time [4]: $\operatorname{Var}\left(x_{n}\right) \equiv\left\langle x_{n}^{2}\right\rangle-\left\langle x_{n}\right\rangle^{2}=4 p q n$, where $x_{n}$ is the displacement of a random walker at the $n$th step, $p$ and $q=1-$ $p$ are the probabilities of right and left jumps, respectively, and we assume the jump size is fixed to unity. Thus, the diffusivity defined by $D \equiv \operatorname{Var}\left(x_{n}\right) / n$ is given by $D=(1-\varepsilon)(1+\varepsilon)$, where $\varepsilon=p-q$. In the absence of a bias $(\varepsilon=0)$ the value of $D$ is maximized. In other words, the diffusivity is always suppressed by the bias for discrete-time random walks.

This trend may in fact be reversed when the time steps are continuous random variables. It is well-known that the diffusivity can be enhanced by an external field for diffusion in periodic potentials, i.e., tilted sinusoidal potentials $[5,6]$. In particular, when the diffusivity in the absence of an external force is small due to deep periodic potential wells or low

\footnotetext{
*takuma@rs.tus.ac.jp
}

Published by the American Physical Society under the terms of the Creative Commons Attribution 4.0 International license. Further distribution of this work must maintain attribution to the author(s) and the published article's title, journal citation, and DOI. temperatures, the diffusivity is greatly accelerated ("giant acceleration") at an optimal external force. As seen above, the external force actually suppresses the diffusivity due to a directed motion in discrete-time random walks. Conversely, the bias decreases the escape time from a valley of a periodic potential, which contributes to the enhancement of diffusivity. With the aid of this trade-off relation, the diffusivity can be maximized at some optimal external force. As the diffusivity enhancement by a bias is a universal phenomenon in diffusion in periodic potentials, many experiments have been designed to realize this effect [7-12].

Effects of a bias in many-body systems has also attracted a considerable interest aiming to unravel nonequilibrium properties [13-16]. In particular, it is essential to investigate the implication of an external force on the diffusion of a particle in many-particle systems $[17,18]$. In many crowding systems, such as diffusion in cells and active diffusion of colloidal particles, diffusion becomes anomalous; i.e., the mean-squared displacement does not increase linearly with time [19-22]. Recently, it became known that dynamics of a biased tracer particle in a crowding environment become anomalous. In particular, an external field in crowding systems induces superdiffusion; i.e., $\left\langle\boldsymbol{r}(t)^{2}\right\rangle \propto t^{\beta}$ with $\beta>1$, where $\boldsymbol{r}(t)$ is a position of a tracer particle [23-28].

The continuous-time random walk (CTRW) is a simple stochastic model of anomalous diffusion and often used as a model of diffusion in heterogeneous environments because it contains many aspects of anomalous transport in disordered media [29-32]. In the CTRW, the time steps of a random walker are continuous random variables and the time between steps is called the waiting time. Effects of a bias in the CTRW have been studied in the context of anomalous transport in porous media [33-35]. In particular, field-induced superdiffusion may be observed when a bias is added in the CTRW [34-38]. This field-induced phenomenon is essential 
to unraveling enhancement of the diffusivity in heterogeneous systems.

In this paper, we investigate an initial-ensemble dependence of the variance of the displacement and ergodic properties of the time-averaged variance of the displacement in the CTRW with drift. When the second moment of the waiting time diverges, the variance becomes superdiffusive. In this regime there is a clear initial-ensemble dependence of statistical quantities such as the correlation function and the mean-squared displacement [39,40], which will give rise to a nonequivalence of time and ensemble averages [40,41]. Therefore, it is a fundamental question of whether the system is still ergodic. If the system is ergodic, it is also important to clarify how the initial ensemble affects the relaxation process.

\section{MODEL}

The quenched trap model (QTM) is used to describe a random walk in a quenched random potential landscape, in which the depths of the potential wells are randomly distributed [42]. A particle will be trapped in a potential well and escape from the well. When the distribution of depths follows an exponential law, the escape-time distribution (we will call it the waiting-time distribution later) becomes of power-law form [43], where the power-law exponent depends on the temperature. CTRW is a random walk with random waiting times, corresponding to an annealed model of the QTM. In CTRW, waiting times are independent and identically distributed (IID) random variables, which do not depend on the position of a particle. On the other hand, the waiting-time distribution clearly depends on the position in the QTM. In this sense, the CTRW is homogeneous and sometimes fails to capture some physical properties in the QTM due to the annealed features [44-48]. However, the CTRW is a good approximation when the spatial dimension is equal and greater than 2 [49] or in the presence of a bias minimizing the risk of back stepping [31].

To investigate the effects of the bias on the diffusive properties in heterogeneous environments, we consider a CTRW with a drift. We assume that the waiting-time distribution follows a power-law distribution

$$
\psi(\tau) \sim \alpha \tau_{0}^{\alpha} \tau^{-1-\alpha} \quad\left(\tau \gg \tau_{0}\right)
$$

The Laplace transform for the case $\alpha>1$ considered here reads

$$
\hat{\psi}(s)= \begin{cases}1-\mu s+c s^{\alpha}+o\left(s^{\alpha}\right) & (1<1, \alpha<2), \\ 1-\mu s+\frac{1}{2}\left(\sigma^{2}+\mu^{2}\right) s^{2}+o\left(s^{2}\right) & (2<\alpha),\end{cases}
$$

where the mean and the variance of the waiting time are denoted by $\mu$ and $\sigma^{2}$, respectively, and $c=|\Gamma(1-\alpha)| \tau_{0}^{\alpha}$. For $\psi(\tau)=\alpha \tau_{0}^{\alpha} \tau^{-1-\alpha}\left(\tau \geqslant \tau_{0}\right), \mu$ and $\sigma^{2}+\mu^{2}$ are given by $\alpha \tau_{0} /(\alpha-1)$ and $\alpha \tau_{0}^{2} /(\alpha-2)$, respectively.

Let $N_{t}$ be the number of jumps of a random walker until time $t$. Then, we have the first moment of displacement $x(t)$ with $x(0)=0$ as

$$
\langle x(t)\rangle=(p-q)\left\langle N_{t}\right\rangle .
$$

The variance of the displacement, $\operatorname{Var}[x(t)] \equiv\left\langle x(t)^{2}\right\rangle-$ $\langle x(t)\rangle^{2}$, is expressed through $N_{t}$ as

$$
\operatorname{Var}[x(t)]=(p-q)^{2}\left(\left\langle N_{t}^{2}\right\rangle-\left\langle N_{t}\right\rangle^{2}\right)+4 p q\left\langle N_{t}\right\rangle .
$$

Moreover, the variance of $\delta x(t, t+\Delta) \equiv x(t+\Delta)-x(t)$, is given by

$$
\begin{aligned}
\operatorname{Var}[ & \delta x(t, t+\Delta)] \\
= & (p-q)^{2}\left(\left\langle N_{t+\Delta}^{2}\right\rangle-\left\langle N_{t}^{2}\right\rangle-2\left\langle N_{t, t+\Delta} N_{t}\right\rangle\right) \\
& -(p-q)^{2}\left(\left\langle N_{t+\Delta}\right\rangle^{2}-\left\langle N_{t}\right\rangle^{2}-2\left\langle N_{t, t+\Delta}\right\rangle\left\langle N_{t}\right\rangle\right) \\
& +4 p q\left(\left\langle N_{t+\Delta}\right\rangle-\left\langle N_{t}\right\rangle\right),
\end{aligned}
$$

where $N_{t, t+\Delta}=N_{t+\Delta}-N_{t}$ is the number of jumps in $[t, t+$ $\Delta]$. Therefore, the mean and variance of the displacement can be calculated using the moments and the correlation function of $N_{t}$, which can be obtained from renewal theory [50].

Here, we consider two typical renewal processes, i.e., ordinary and equilibrium renewal processes [50]. Renewal processes are point processes in which the time intervals between successive renewals are IID random variables. Because the waiting times are IID random variables in the CTRW, statistical properties of CTRW can be obtained using the renewal processes [51]. In CTRW, the distribution of the time intervals between successive renewals corresponds to the waiting-time distribution $\psi(\tau)$.

One has to be careful about the first renewal event because the distribution of the first renewal time is not the same as $\psi(\tau)$ in general [50-52]. An ordinary renewal process is a renewal process in which the distribution of the time when the first renewal occurs follows $\psi(\tau)$ [50]. In other words, a renewal occurs at the time when the observation starts. In equilibrium renewal processes, a measurement starts after the system has evolved for a long time, and thus the distribution of the first renewal time is not the same as $\psi(\tau)$, except for the case when the waiting-time distribution follows an exponential law. When the mean waiting time exists $(\mu<\infty)$, the distribution of the first renewal time is given by $[50,52]$

$$
\psi_{0}(\tau)=\mu^{-1} \int_{\tau}^{\infty} \psi\left(\tau^{\prime}\right) d \tau^{\prime}
$$

\section{ENSEMBLE-AVERAGED VARIANCE}

The first moment $\left\langle N_{t}\right\rangle$, called the renewal function, has a well-known expression in renewal theory [50,52]: for $\alpha>1$ it becomes $\left\langle N_{t}\right\rangle \sim t / \mu$ for $t \gg \tau_{0}$. In particular, it is exact; i.e., $\left\langle N_{t}\right\rangle=t / \mu$ for $t>0$, when the first renewal time follows the equilibrium distribution (6). Using Eq. (3) and the renewal function, we have the mean displacements,

$$
\langle x(t)-x(0)\rangle_{\mathrm{eq}}=(\varepsilon / \mu) t
$$

for $t>0$ and

$$
\langle x(t)-x(0)\rangle_{\text {or }} \sim(\varepsilon / \mu) t
$$

for $t \gg \tau_{0}$ in equilibrium $\left(\langle\cdot\rangle_{\mathrm{eq}}\right)$ and ordinary $\left(\langle\cdot\rangle_{\mathrm{or}}\right)$ renewal processes, respectively. Therefore, the flow velocity $c_{\varepsilon}$ is given by $c_{\varepsilon}=\varepsilon / \mu$ for both types of renewal processes.

The second moment of $N_{t}$ is also well-known in renewal theory [50]. For $\alpha>2$, the asymptotic behavior of the variance of $N_{t}$ is not affected by the initial ensemble and is given by

$$
\operatorname{Var}\left(N_{t}\right) \equiv\left\langle N_{t}^{2}\right\rangle-\left\langle N_{t}\right\rangle^{2}=\frac{\sigma^{2}}{\mu^{3}} t+o(t) .
$$




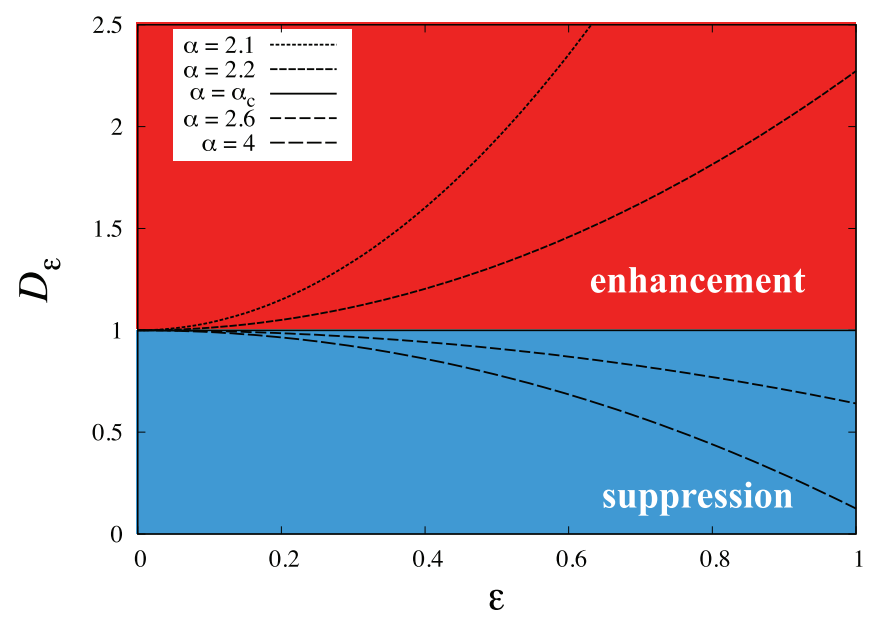

FIG. 1. Effect of bias $\varepsilon$ on diffusivity for different $\alpha$, where the mean is set to unity $(\mu=1)$. Solid and dashed curves are the theoretical results, Eq. (16), i.e., $D=1+\left(\sigma^{2}-1\right) \varepsilon^{2}$. The diffusivity can be enhanced by the bias for $\alpha>\alpha_{c}$.

Therefore, the variance of the displacement for both ordinary and equilibrium processes becomes

$$
\operatorname{Var}[x(t)] \sim\left(\frac{1}{\mu}+\frac{\sigma^{2}-\mu^{2}}{\mu^{3}} \varepsilon^{2}\right) t
$$

for $t \gg \tau_{0}$ and $\alpha>2$. The diffusion coefficient given by

$$
D_{\varepsilon} \equiv \frac{1}{\mu}+\frac{\sigma^{2}-\mu^{2}}{\mu^{3}} \varepsilon^{2}
$$

is increased by the bias $\varepsilon$ when $\sigma^{2}>\mu^{2}$. The critical value $\alpha_{c}$, i.e., $\sigma^{2}=\mu^{2}$ at $\alpha=\alpha_{c}$, is given by $\alpha_{c}=1+\sqrt{2}$. Therefore, the diffusion is enhanced when $2<\alpha<\alpha_{c}$ (Fig. 1). When the variance $\sigma^{2}$ of the waiting time is greater than $\mu^{2}$, an enhancement of the diffusivity due to the external force is achieved. In a general situation, the diffusion coefficient was already derived in Refs. [30,35]. We note that this enhancement mechanism is completely different from that for diffusion in tilted periodic potentials $[5,6]$.

The ratio between $c_{\varepsilon}$ and $D_{\varepsilon}$ becomes

$$
\frac{c_{\varepsilon}}{D_{\varepsilon}}=\frac{\varepsilon}{1+\left(\sigma^{2} / \mu^{2}-1\right) \varepsilon^{2}} .
$$

It is an increasing function of $\varepsilon$ on [0,1] for $\sigma^{2}<2 \mu^{2}$, i.e., $\alpha>\alpha^{*} \equiv 1+\sqrt{6} / 2$. Conversely, it has a peak at $\varepsilon^{*}=$ $\mu / \sqrt{\sigma^{2}-\mu^{2}}$ for $2<\alpha<\alpha^{*}$.

To obtain $\operatorname{Var}[\delta x(t, t+\Delta)]$, we need to calculate the correlation function $\left\langle N_{t} N_{t, t+\Delta}\right\rangle$. Following Ref. [53], one can obtain the Laplace transform of $\left\langle N_{t} N_{t, t+\Delta}\right\rangle$ with respect to $t$ and $\Delta$ as

$$
\underset{u, s}{\mathcal{L}}\left\langle N_{t} N_{t, t+\Delta}\right\rangle_{\text {eq }}=\frac{\hat{\psi}(s)-\hat{\psi}(u)}{\mu u s(u-s)[1-\hat{\psi}(u)][1-\hat{\psi}(s)]}
$$

and

$$
\underset{u, s}{\mathcal{L}}\left\langle N_{t} N_{t, t+\Delta}\right\rangle_{\text {or }}=\frac{[\hat{\psi}(s)-\hat{\psi}(u)] \hat{\psi}(u)}{s(u-s)[1-\hat{\psi}(u)]^{2}[1-\hat{\psi}(s)]} .
$$

By the inverse Laplace transform, we have

$$
\left\langle N_{t} N_{t, t+\Delta}\right\rangle \sim \frac{t \Delta}{\mu^{2}}+\frac{\left(\sigma^{2}-\mu^{2}\right) \Delta}{2 \mu^{3}}+o(\Delta)
$$

for $\alpha>2$ and $\tau_{0} \ll \Delta \ll t$, which is valid for both types of renewal processes. Therefore, the variance

$$
\operatorname{Var}[\delta x(t, t+\Delta)] \sim \varepsilon^{2} \frac{\sigma^{2}}{\mu^{3}} \Delta+\left(1-\varepsilon^{2}\right) \frac{\Delta}{\mu}=\operatorname{Var}\left(x_{\Delta}\right)
$$

is stationary, i.e., independent of $t$ in the asymptotic limit.

Unlike the asymptotic behavior of the variance for $\alpha>2$, interesting aging effects are observed for $1<\alpha<2$ [54]. In fact, we obtain the following initial-ensemble dependence:

$$
\left\langle N_{t}^{2}\right\rangle_{\text {or }}-\left\langle N_{t}\right\rangle_{\text {or }}^{2}=(\alpha-1) D(\alpha) t^{3-\alpha}+o\left(t^{3-\alpha}\right),
$$

and

$$
\left\langle N_{t}^{2}\right\rangle_{\mathrm{eq}}-\left\langle N_{t}\right\rangle_{\mathrm{eq}}^{2}=D(\alpha) t^{3-\alpha}+o\left(t^{3-\alpha}\right),
$$

where $D(\alpha)=2 c \mu^{-3} / \Gamma(4-\alpha)$. It follows that the variances of the displacement for ordinary and equilibrium renewal processes become

$$
\operatorname{Var}[x(t)]_{\mathrm{or}}=\varepsilon^{2}(\alpha-1) D(\alpha) t^{3-\alpha}+4 p q \frac{t}{\mu}+o(t)
$$

and

$$
\operatorname{Var}[x(t)]_{\mathrm{eq}}=\varepsilon^{2} D(\alpha) t^{3-\alpha}+4 p q \frac{t}{\mu}+o(t),
$$

respectively. Therefore, the spreading of particles with respect to the mean in the regime $1<\alpha<2$ becomes superdiffusive with exponent $(3-\alpha)$. We note that the coefficients of the leading terms differ by a factor $(\alpha-1)$ according to the initial ensemble. This initial-ensemble dependence is sometimes observed when the second moment of the waiting time diverges [39-41,55].

For $1<\alpha<2$ and $\Delta \ll t$, the correlation function $\left\langle N_{t} N_{t, t+\Delta}\right\rangle$ becomes

$$
\begin{aligned}
\left\langle N_{t} N_{t, t+\Delta}\right\rangle_{\text {or }} \sim & \frac{t \Delta}{\mu^{2}}+\frac{2 c t^{2-\alpha} \Delta}{\mu^{3} \Gamma(3-\alpha)}-\frac{c \Delta^{3-\alpha}}{\mu^{3} \Gamma(4-\alpha)} \\
& +\frac{c t^{1-\alpha} \Delta^{2}}{2 \mu^{3} \Gamma(2-\alpha)}+o\left(t^{1-\alpha} \Delta^{2}\right) .
\end{aligned}
$$

It follows that for the ordinary renewal process the variance of $\delta x(t, t+\Delta)$ with $t \gg \Delta$ becomes

$$
\begin{aligned}
\operatorname{Var}[\delta x(t, t+\Delta)]_{\mathrm{or}} \sim & \varepsilon^{2} D(\alpha) \Delta^{3-\alpha}-\frac{\varepsilon^{2} c \Delta^{2}}{\mu^{3} \Gamma(2-\alpha) t^{\alpha-1}} \\
& +4 p q\left(\frac{\Delta}{\mu}+\frac{(2-\alpha) c \Delta}{\mu^{2} \Gamma(3-\alpha) t^{\alpha-1}}\right) .
\end{aligned}
$$

Therefore, the variance of $\delta x(t, t+\Delta)$ for the ordinary renewal process has a clear $t$ dependence and approaches that of the equilibrium renewal process (see Fig. 2). Although the dependence on the aging time $t$ gradually disappears, i.e., $\operatorname{Var}[\delta x(t, t+\Delta)]_{\text {or }} \rightarrow \operatorname{Var}[x(\Delta)]_{\text {eq }}$ for $t \rightarrow \infty$, the aging effect lasts for a long time when $\alpha$ is close to unity. This recovery 


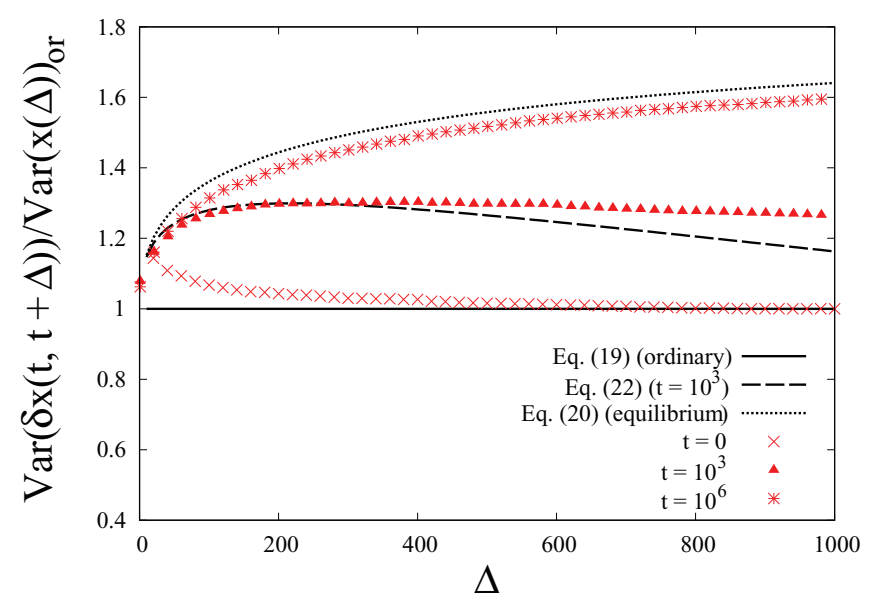

FIG. 2. Ensemble-averaged variance of $\delta x(t, t+\Delta)$ divided by $\operatorname{Var}[x(\Delta)]_{\text {or }}$ for different aging times $t(\alpha=1.5)$. Symbols: numerical simulations, in which we generated waiting times according to the PDF $\psi(\tau)=\alpha \tau^{-1-\alpha}(\tau \geqslant 1)$. Solid lines: Eqs. (19) and (22). The variance increases with growing $t$ and approaches that of the equilibrium renewal process, Eq. (20).

of diffusivity (rejuvenation of diffusivity) is achieved at a longer aging time. In other words, if one waits to start measurements for a long time, the observed diffusivity approaches that of the equilibrium initial ensemble, which is enhanced by the factor $(\alpha-1)^{-1}$. This enhancement becomes significant especially for $\alpha \rightarrow 1$.

\section{TIME-AVERAGED VARIANCE}

Here, we define the time-averaged variance (TAV) of the displacement as

$$
\overline{\operatorname{Var}\left(x_{\Delta} ; t\right)}=\frac{1}{t-\Delta} \int_{0}^{t-\Delta}\left(\delta x\left(t^{\prime}, t^{\prime}+\Delta\right)-\frac{\varepsilon \Delta}{\mu}\right)^{2} d t^{\prime},
$$

where $\varepsilon \Delta / \mu=\left\langle\delta x\left(t^{\prime}, t^{\prime}+\Delta\right)\right\rangle_{\mathrm{eq}}$, which does not depend on $t^{\prime}$. The variable $t$ in Eq. (23) now corresponds to the total measurement time, $\Delta$ is the lag time, and the overline denotes time averaging. Expanding the integrand, the TAV can be written as

$$
\begin{aligned}
\overline{\operatorname{Var}\left(x_{\Delta} ; t\right)}-\left(\frac{\Delta}{\mu}\right)^{2} \varepsilon^{2}= & \frac{1}{t-\Delta} \int_{0}^{t-\Delta} \delta x\left(t^{\prime}, t^{\prime}+\Delta\right)^{2} d t^{\prime} \\
& -\frac{2 \varepsilon \Delta}{\mu(t-\Delta)} \int_{0}^{t-\Delta} \delta x\left(t^{\prime}, t^{\prime}+\Delta\right) d t^{\prime}
\end{aligned}
$$

As follows from Eqs. (16) and (22), the ensemble average of the TAV converges to a constant for $\alpha>1$,

$$
\left\langle\overline{\operatorname{Var}\left(x_{\Delta} ; t\right)}\right\rangle \rightarrow \operatorname{Var}\langle x(\Delta)\rangle_{\mathrm{eq}}
$$

as $t \rightarrow \infty$. In particular, the ensemble average of the TAV for the ordinary renewal process becomes

$$
\left\langle\overline{\operatorname{Var}\left(x_{\Delta} ; t\right)}\right\rangle_{\text {or }}-\operatorname{Var}\langle x(\Delta)\rangle_{\mathrm{eq}} \sim K(\alpha) t^{1-\alpha}
$$

for $t \rightarrow \infty$, where $K(\alpha)=\frac{\varepsilon^{2} c \Delta^{2}}{\mu^{3} \Gamma(2-\alpha)}\left(\frac{\mu}{\varepsilon^{2} \Delta}-1\right)$ for $1<\alpha<2$. Thus, the convergence to $\operatorname{Var}[x(\Delta)]_{\mathrm{eq}}$ becomes significantly slower for $\alpha \cong 1$.

The time average of the displacement can be approximated by $\int_{0}^{t-\Delta} \delta x\left(t^{\prime}, t^{\prime}+\Delta\right) d t^{\prime} /(t-\Delta) \sim \sum_{k=1}^{N_{t}} z_{k} \Delta / t$ for $t \gg \Delta$, where $z_{k}$ is the $k$ th jump $\left(z_{k}= \pm 1\right)$. By the law of large numbers, i.e., $\sum_{k=1}^{n} z_{k} / n \rightarrow\left\langle z_{k}\right\rangle=\varepsilon$ for $n \rightarrow \infty$, we have

$$
\frac{1}{t-\Delta} \int_{0}^{t-\Delta} \delta x\left(t^{\prime}, t^{\prime}+\Delta\right) d t^{\prime} \sim \frac{N_{t}}{t} \varepsilon \Delta .
$$

Here, we use a similar approximation for the squared displacements invented in Ref. [56] (see also the argument in Refs. [51,57]). While this approximation is used for the CTRW without bias, it is also valid for the CTRW with bias [57]. Therefore, we have

$$
\frac{1}{t-\Delta} \int_{0}^{t-\Delta} \delta x\left(t^{\prime}, t^{\prime}+\Delta\right)^{2} d t^{\prime} \sim \frac{N_{t}}{t}\left[\Delta+\varepsilon^{2} h(\Delta)\right],
$$

where $h(\Delta)$ is a function of $\Delta$. For $\alpha>1(\mu<\infty)$, the TAV becomes

$$
\overline{\operatorname{Var}\left(x_{\Delta} ; t\right)}-\varepsilon^{2}\left(\frac{\Delta}{\mu}\right)^{2} \sim \frac{N_{t}}{t} H(\Delta),
$$

where $H(\Delta)=\Delta+\varepsilon^{2} h(\Delta)-\frac{2 \varepsilon^{2} \Delta^{2}}{\mu}$. Taking the ensemble average of Eq. (29) and using Eq. (25) lead to $h(\Delta)=$ $\frac{\sigma^{2}-\mu^{2}}{\mu^{2}} \Delta+\frac{\Delta^{2}}{\mu}$ and $h(\Delta)=\mu D(\alpha) \Delta^{3-\alpha}-\Delta+\frac{\Delta^{2}}{\mu}$ for $\alpha>2$ and $1<\alpha<2$, respectively. We confirmed numerically that this relation is valid only for $\alpha<2$ (results not shown here). For $\alpha>2$, the ensemble average of $H(\Delta)$ is indeed given by $\langle H(\Delta)\rangle=\Delta+\varepsilon^{2} h(\Delta)-\frac{2 \varepsilon^{2} \Delta^{2}}{\mu}$ but deviations of $H(\Delta)$ from $\langle H(\Delta)\rangle$ cannot be ignored, and will be considered in detail elsewhere.

To characterize the relaxation process, we consider the relative standard deviation (RSD) [58] of the TAV

$$
\Sigma(t ; \Delta) \equiv \frac{\sqrt{\left\langle\left\{\overline{\operatorname{Var}\left(x_{\Delta} ; t\right)}\right\}^{2}\right\rangle-\left\langle\overline{\operatorname{Var}\left(x_{\Delta} ; t\right)}\right\rangle^{2}}}{\left\langle\overline{\operatorname{Var}\left(x_{\Delta} ; t\right)}\right\rangle} .
$$

This is the squared root of the ergodicity breaking parameter, which is widely used to investigate ergodic properties [57,59]. For $1<\alpha<2$ using Eq. (29) we have

$$
\Sigma(t ; \Delta) \sim \sqrt{\frac{\left\langle N_{t}^{2}\right\rangle-\left\langle N_{t}\right\rangle^{2}}{t^{2}}} \frac{|H(\Delta)|}{\operatorname{Var}\left(x_{\Delta}\right)} .
$$

Therefore, the RSD for $\tau_{0} \ll \Delta \ll t$ becomes

$$
\Sigma(t ; \Delta) \sim \sqrt{D(\alpha)}\left|\mu-\frac{\varepsilon^{2} \Delta^{2}}{\mu \operatorname{Var}\left(x_{\Delta}\right)}\right| t^{-\frac{\alpha-1}{2}}
$$

for the equilibrium initial ensemble. For $1<\alpha<2$, the RSD decays as $t^{-\frac{\alpha-1}{2}}$, which is anomalously slower than the usual case, $t^{-\frac{1}{2}}$ [59]. Therefore, trajectory-to-trajectory fluctuations of the TAV remain large even for long measurement times. For CTRW with $1<\alpha<2$, similar anomalies are ubiquitous in time-averaged observables $[60,61]$.

\section{CONCLUSION}

The diffusivity for diffusion processes with drift is characterized by the variance of the particle displacement. In the 
paradigmatic CTRW model, a bias plays a significant role in the enhancement of diffusion, which is supported by an increased variance of waiting times. In particular, the diffusivity becomes infinite (superdiffusive) when the variance diverges. In the superdiffusive regime, we found an intrinsic difference of the diffusivity due to the initial ensembles, e.g., for ordinary and equilibrium renewal processes. For the ordinary renewal process, we uncovered that the diffusivity increases approaching that of the corresponding equilibrium process for a long aging time. If one allows the system to age before start of the measurement, for a longer aging time the diffusivity is found to be greatly enhanced compared with that in the absence of aging, especially when the exponent $\alpha$ is close to unity. This recovering of diffusivity has a significant implication of rejuvenation in superdiffusive physical systems. We also showed that TAVs converge to a constant, which is given by the ensemble-averaged variance with the equilibrium initial ensemble. Therefore, the system is ergodic, whereas there is a distinct dependence of the ensemble-averaged variance on the initial ensemble. Finally, we found that trajectory-totrajectory fluctuations of the TAVs decay anomalously slowly, as compared to standard random walks.

\section{ACKNOWLEDGMENTS}

T.A. was supported by the Grant-in-Aid for Scientific Research (B) of the JSPS (Grant No. 16KT0021). Support from DFG in projects ME 1535/6-1 and ME 1535/7-1 as well as from the Foundation for Polish Science (Humboldt Polish Honorary Research Scholarship) is gratefully acknowledged.
[1] J. B. Knight, A. Vishwanath, J. P. Brody, and R. H. Austin, Phys. Rev. Lett. 80, 3863 (1998).

[2] T. M. Squires and S. R. Quake, Rev. Mod. Phys. 77, 977 (2005).

[3] C. Y. Lim, Y. C. Lam, and C. Yang, Biomicrofluidics 4, 014101 (2010).

[4] W. Feller, An Introduction to Probability Theory and Its Applications (Wiley, New York, 1968), Vol. 1.

[5] P. Reimann, C. Van den Broeck, H. Linke, P. Hänggi, J. M. Rubi, and A. Pérez-Madrid, Phys. Rev. Lett. 87, 010602 (2001).

[6] P. Reimann, C. Van den Broeck, H. Linke, P. Hänggi, J. M. Rubi, and A. Pérez-Madrid, Phys. Rev. E 65, 031104 (2002).

[7] M. Evstigneev, O. Zvyagolskaya, S. Bleil, R. Eichhorn, C. Bechinger, and P. Reimann, Phys. Rev. E 77, 041107 (2008).

[8] P. Reimann and R. Eichhorn, Phys. Rev. Lett. 101, 180601 (2008).

[9] R. Hayashi, K. Sasaki, S. Nakamura, S. Kudo, Y. Inoue, H. Noji, and K. Hayashi, Phys. Rev. Lett. 114, 248101 (2015).

[10] X.-G. Ma, P.-Y. Lai, B. J. Ackerson, and P. Tong, Phys. Rev. E 91, 042306 (2015).

[11] D. Kim, C. Bowman, J. T. Del Bonis-O’Donnell, A. Matzavinos, and D. Stein, Phys. Rev. Lett. 118, 048002 (2017).

[12] X.-G. Ma, Y. Su, P.-Y. Lai, and P. Tong, Phys. Rev. E 96, 012601 (2017).

[13] P. Habdas, D. Schaar, A. C. Levitt, and E. R. Weeks, Europhys. Lett. 67, 477 (2004).

[14] C. Wilhelm, Phys. Rev. Lett. 101, 028101 (2008).

[15] I. Gazuz, A. M. Puertas, T. Voigtmann, and M. Fuchs, Phys. Rev. Lett. 102, 248302 (2009).

[16] J. Shin, A. G. Cherstvy, W. K. Kim, and R. Metzler, New J. Phys. 17, 113008 (2015).

[17] C. J. Harrer, D. Winter, J. Horbach, M. Fuchs, and T. Voigtmann, J. Phys.: Cond. Matt. 24, 464105 (2012).

[18] M. Gruber, G. C. Abade, A. M. Puertas, and M. Fuchs, Phys. Rev. E 94, 042602 (2016).

[19] R. Richert, J. Phys.: Cond. Matt. 14, R703 (2002).

[20] I. Golding and E. C. Cox, Phys. Rev. Lett. 96, 098102 (2006).

[21] A. Weigel, B. Simon, M. Tamkun, and D. Krapf, Proc. Natl. Acad. Sci. USA 108, 6438 (2011).

[22] F. Höfling and T. Franosch, Rep. Prog. Phys. 76, 046602 (2013); K. Nørregaard, R. Metzler, C. M. Ritter, K. Berg-Sørensen, and L. B. Oddershede, Chem. Rev. 117, 4342 (2017);
[23] C. F. E. Schroer and A. Heuer, Phys. Rev. Lett. 110, 067801 (2013).

[24] O. Bénichou, A. Bodrova, D. Chakraborty, P. Illien, A. Law, C. Mejía-Monasterio, G. Oshanin, and R. Voituriez, Phys. Rev. Lett. 111, 260601 (2013).

[25] O. Bénichou, P. Illien, C. Mejía-Monasterio, and G. Oshanin, J. Stat. Mech. (2013) P05008.

[26] J. F. Reverey, J.-H. Jeon, H. Bao, M. Leippe, R. Metzler, and C. Selhuber-Unkel, Sci. Rep. 5, 11690 (2015).

[27] G. Gradenigo, E. Bertin, and G. Biroli, Phys. Rev. E 93, 060105 (2016).

[28] S. Leitmann and T. Franosch, Phys. Rev. Lett. 118, 018001 (2017).

[29] E. W. Montroll and G. H. Weiss, J. Math. Phys. 6, 167 (1965).

[30] M. F. Shlesinger, J. Stat. Phys. 10, 421 (1974).

[31] H. Scher and E. W. Montroll, Phys. Rev. B 12, 2455 (1975).

[32] R. Metzler and J. Klafter, Phys. Rep. 339, 1 (2000).

[33] H. Scher, G. Margolin, and B. Berkowitz, Chem. Phys. 284, 349 (2002).

[34] G. Margolin and B. Berkowitz, Phys. Rev. E 65, 031101 (2002).

[35] B. Berkowitz, A. Cortis, M. Dentz, and H. Scher, Rev. Geophys. 44 (2006).

[36] R. Burioni, G. Gradenigo, A. Sarracino, A. Vezzani, and A. Vulpiani, J. Stat. Mech. (2013) P09022.

[37] R. Burioni, G. Gradenigo, A. Sarracino, A. Vezzani, and A. Vulpiani, Commun. Theor. Phys. 62, 514 (2014).

[38] R. Hou, A. G. Cherstvy, R. Metzler, and T. Akimoto, Phys. Chem. Chem. Phys. (2018), doi: 10.1039/C8CP01863D

[39] T. Akimoto and Y. Aizawa, J. Korean Phys. Soc. 50, 254 (2007).

[40] T. Akimoto, Phys. Rev. Lett. 108, 164101 (2012).

[41] A. Godec and R. Metzler, Phys. Rev. Lett. 110, 020603 (2013); Phys. Rev. E 88, 012116 (2013).

[42] J.-P. Bouchaud and A. Georges, Phys. Rep. 195, 127 (1990).

[43] F. Bardou, J.-P. Bouchaud, A. Aspect, and C. Cohen-Tannoudji, Levy Statistics and Laser Cooling: How Rare Events Bring Atoms to Rest (Cambridge University, New York, 2002).

[44] E. M. Bertin and J.-P. Bouchaud, Phys. Rev. E 67, 026128 (2003).

[45] S. Burov and E. Barkai, Phys. Rev. Lett. 98, 250601 (2007).

[46] T. Miyaguchi and T. Akimoto, Phys. Rev. E 83, 031926 (2011). 91, 010102 (2015).

[47] L. Luo and L.-H. Tang, Phys. Rev. E 92, 042137 (2015). 
[48] T. Akimoto, E. Barkai, and K. Saito, Phys. Rev. Lett. 117, 180602 (2016); Phys. Rev. E 97, 052143 (2018).

[49] J. Machta, J. Phys. A 18, L531 (1985).

[50] D. R. Cox, Renewal Theory (Methuen, London, 1962).

[51] J. H. P. Schulz, E. Barkai, and R. Metzler, Phys. Rev. Lett. 110, 020602 (2013); Phys. Rev. X 4, 011028 (2014).

[52] C. Godrèche and J. M. Luck, J. Stat. Phys. 104, 489 (2001).

[53] E. Barkai, Phys. Rev. E 75, 060104 (2007).

[54] P. Allegrini, J. Bellazzini, G. Bramanti, M. Ignaccolo, P. Grigolini, and J. Yang, Phys. Rev. E 66, 015101 (2002).

[55] D. Froemberg and E. Barkai, Phys. Rev. E 87, 030104 (2013).
[56] T. Miyaguchi and T. Akimoto, Phys. Rev. E 87, 032130 (2013).

[57] Y. He, S. Burov, R. Metzler, and E. Barkai, Phys. Rev. Lett. 101, 058101 (2008).

[58] T. Uneyama, T. Akimoto, and T. Miyaguchi, J. Chem. Phys. 137, 114903 (2012); T. Uneyama, T. Miyaguchi, and T. Akimoto, Phys. Rev. E 92, 032140 (2015).

[59] R. Metzler, J.-H. Jeon, A. G. Cherstvy, and E. Barkai, Phys. Chem. Chem. Phys. 16, 24128 (2014).

[60] T. Akimoto, E. Yamamoto, K. Yasuoka, Y. Hirano, and M. Yasui, Phys. Rev. Lett. 107, 178103 (2011).

[61] J. H. P. Schulz and E. Barkai, Phys. Rev. E 91, 062129 (2015). 